\title{
Variation in the management of isolated craniosynostosis: a survey of the Synostosis Research Group
}

\author{
John R. W. Kestle, MD, ${ }^{1}$ Amy Lee, MD, ${ }^{2}$ Richard C. E. Anderson, MD, ${ }^{3}$ Barbu Gociman, MD, ${ }^{4}$ \\ Kamlesh B. Patel, MD, MSc, ${ }^{5}$ Matthew D. Smyth, MD, ${ }^{6}$ Craig Birgfeld, MD, ${ }^{7}$ lan F. Pollack, MD, ${ }^{8}$ \\ Jesse A. Goldstein, MD, ${ }^{9}$ Mandeep Tamber, MD, PhD, ${ }^{8}$ Thomas Imahiyerobo, MD, ${ }^{10}$ and \\ Faizi A. Siddiqi, MD, ${ }^{4}$ for the Synostosis Research Group
}

1Department of Neurosurgery, Division of Pediatric Neurosurgery, Primary Children's Hospital, University of Utah, Salt Lake City, Utah; Departments of ${ }^{2}$ Neurological Surgery and ${ }^{7}$ Surgery, Division of Plastic Surgery, Seattle Children's Hospital, University of Washington, Seattle, Washington; 'Department of Neurological Surgery, Columbia University, Morgan Stanley Children's Hospital of NewYork-Presbyterian, New York, New York; ${ }^{4}$ Division of Plastic Surgery and Reconstructive Surgery, University of Utah, Salt Lake City, Utah; ${ }^{5}$ Division of Plastic and Reconstructive Surgery, Department of Surgery, and ${ }^{6}$ Department of Neurosurgery, St. Louis Children's Hospital, Washington University School of Medicine in St. Louis, Missouri; Departments of ${ }^{8}$ Pediatric Neurosurgery and ${ }^{9}$ Plastic Surgery, Children's Hospital of Pittsburgh of UPMC, Pittsburgh, Pennsylvania; and ${ }^{10}$ Division of Plastic Surgery, Columbia University Medical Center, NewYork-Presbyterian Hospital, New York, New York

OBJECTIVE The authors created a collaborative network, the Synostosis Research Group (SynRG), to facilitate multicenter clinical research on craniosynostosis. To identify common and differing practice patterns within the network, they assessed the SynRG surgeons' management preferences for sagittal synostosis. These results will be incorporated into planning cooperative studies.

METHODS The SynRG consists of 12 surgeons at 5 clinical sites. An email survey was distributed to SynRG surgeons in late 2016, and responses were collected through early 2017. Responses were collated and analyzed descriptively.

RESULTS All of the surgeons-7 plastic/craniofacial surgeons and 5 neurosurgeons-completed the survey. They varied in both experience (1-24 years) and sagittal synostosis case volume in the preceding year (5-45 cases). Three sites routinely perform preoperative CT scans. The preferred surgical technique for children younger than 3 months is strip craniectomy (10/12 surgeons), whereas children older than 6 months are all treated with open cranial vault surgery. Pre-incision cefazolin, preoperative complete blood count panels, and an arterial line were used by most surgeons, but tranexamic acid was used routinely at 3 sites and never at the other 2 sites. Among surgeons performing endoscopic strip craniectomy surgery (SCS), most create a 5-cm-wide craniectomy, whereas 2 surgeons create a 2-cm strip. Four surgeons routinely send endoscopic SCS patients to the intensive care unit after surgery. Two of the 5 sites routinely obtain a CT scan within the 1st year after surgery.

CONCLUSIONS The SynRG surgeons vary substantially in the use of imaging, the choice of surgical procedure and technique, and follow-up. A collaborative network will provide the opportunity to study different practice patterns, reduce variation, and contribute multicenter data on the management of children with craniosynostosis.

https://thejns.org/doi/abs/10.3171/2018.7.PEDS18132

KEYWORDS craniosynostosis; sagittal synostosis; network; clinical research; survey; strip craniectomy surgery; cranial vault surgery; craniofacial

$\mathrm{C}$ OLLABORATIVE clinical research networks have been effective in many areas of medicine. They have the ability to accumulate large amounts of data, demonstrate practice variation, answer important clinical questions, and enhance the level of evidence in the litera- ture. Because of these successes, we set out to establish a multicenter collaborative research network to study craniosynostosis. The Synostosis Research Group (SynRG) was formulated in 2016 and began collecting data in the spring of 2017. At present, the SynRG consists of 5 sites with at

ABBREVIATIONS SCS = strip craniectomy surgery; SynRG = Synostosis Research Group.

SUBMITTED February 28, 2018. ACCEPTED July 11, 2018.

INCLUDE WHEN CITING Published online September 14, 2018; DOI: 10.3171/2018.7.PEDS18132. 
TABLE 1. Surgical volume and percentage of endoscopic strip craniectomy procedures for isolated sagittal synostosis

\begin{tabular}{ccc}
\hline Site & Annual No. of New Patients & SCSs (\%) \\
\hline A & 13 & 67 \\
\hline B & 20 & $25^{*}$ \\
\hline C & 30 & 67 \\
\hline D & 35 & 83 \\
\hline E & 45 & 20 \\
\hline
\end{tabular}

* Strip craniectomy at site B was an open strip craniectomy with the placement of springs. All strip procedures at other sites were endoscope-assisted craniectomy with postoperative helmet therapy.

least one neurosurgeon and one plastic surgeon at each site. The sites use a common database to prospectively capture detailed information on each child encountered with a diagnosis of craniosynostosis. As part of the launch of this network, we surveyed SynRG surgeons to identify areas of practice that we have in common and areas in which we differ. This will help us identify topics for further study and assess the feasibility of developing common protocols. We chose sagittal synostosis for this survey as it is the most common form of craniosynostosis and its methods of management are well described. Using a survey of participating surgeons, we sought to discern areas of commonality and variation in practice within the SynRG.

\section{Methods}

A questionnaire was designed to assess surgeon and practice profiles, methods of patient assessment, use of imaging, surgical technique, and follow-up management. The survey was distributed via email to all 12 surgeons in the SynRG. Unclear responses were clarified by telephone call or email. Data were collated in Excel and analyzed descriptively using frequencies, means, and proportions. Some responses were uniform across surgeons at the same site, but others were not. Data are presented in either format as appropriate to the question.

\section{Results}

\section{Surgeon and Practice Profile}

The survey was distributed to all 12 surgeons in late 2016. Responses were collected through early 2017, and all 12 surgeons responded. Seven surgeons had completed residency in plastic surgery and 5 in neurosurgery. The plastic surgeons all completed fellowship training in craniofacial surgery, and the neurosurgeons all completed fellowship training in pediatric neurosurgery. All the surgeons had been in practice from 1 to 24 years (mean 9.9 years). There were 2 professors, 4 associate professors, 5 assistant professors, and 1 instructor. All of the surgeons participate in research activities, and 6 had formal training in research methods.

In the year prior to the survey, the volume of surgically treated isolated sagittal synostosis cases varied among the surgeons from 5 to 45 cases per year. The percentage of cases treated via strip craniectomy surgery (SCS) by each surgeon ranged from $0 \%$ to $88 \%$ (mean $47 \%$ ), suggesting
TABLE 2. Steps involved in new patient assessment for isolated sagittal synostosis

\begin{tabular}{lcc}
\hline \multicolumn{1}{c}{ Step } & $\begin{array}{c}\text { No. of Sites } \\
(\mathrm{n}=5)\end{array}$ & $\begin{array}{c}\text { No. of Surgeons } \\
(\mathrm{n}=12)\end{array}$ \\
\hline Patient sees the whole team at once & 3 & \\
\hline Document & & 12 \\
\hline Family history & & 12 \\
\hline Developmental history & 8 \\
\hline Occipital frontal circumference & & \\
\hline Cephalic index & & \\
\hline Neurological exam & & \\
\hline Imaging & 3 & \\
\hline Preop CT & 4 & \\
\hline 2D photos & 1 & \\
\hline 3D photos & 1 \\
\hline Laser scan & \\
\hline Consults & 1 \\
\hline Genetics & 1 \\
\hline Ophthalmology & \\
\hline $\mathrm{n}=$ total number.
\end{tabular}

substantial variation in surgical approach. The method of treatment was not strongly associated with surgical volume (Table 1).

\section{Initial Evaluation of New Patients}

Clinic models for patient assessment differed. At 3 of the 5 sites, the initial evaluation of new patients is done by the plastic surgeon and neurosurgeon together (with or without a clinic coordinator, nurse, residents, students, social worker); at the other 2 sites, the patient sees the neurosurgical team and the plastic surgery team separately.

The survey asked about routine initial patient assessments (Table 2). In addition to the routine history and physical examination, all surgeons collect family and developmental history, and most (8/12) measure the head circumference. Measurement of the cephalic index with calipers is rare (3/12). A neurological examination is performed by all of the neurosurgeons and one of the plastic surgeons.

The use of CT scanning at the initial evaluation is uniform within each site, and 3/5 sites routinely perform a CT scan that has not already been done. Four sites also use two-dimensional photographs, one uses three-dimensional photographs, one uses laser scans, and none use MRI. Requests for consultation from genetics are part of the routine initial evaluation at one site, and assessment by ophthalmology (with visual evoked potential testing) is routinely done at one other site.

Surgeons were asked about their preoperative discussions with patients and families (Table 3). If a child is younger than 3 months, all surgeons discuss both SCS and open cranial vault surgery as treatment options. As children present at an older age, the preoperative discussion shifts toward open surgery. For children 3-6 months of age at the initial evaluation, 9 surgeons still discuss both surgical techniques. For children older than 6 months, 
TABLE 3. Surgical options for isolated sagittal synostosis by patient age

\begin{tabular}{|c|c|}
\hline Option & No. of Surgeons $(n=12)$ \\
\hline \multicolumn{2}{|c|}{$\begin{array}{l}\text { SCS option discussed w/ family of child } \\
\text { aged }\end{array}$} \\
\hline$<3$ mos old & 12 \\
\hline $3-6$ mos old & 9 \\
\hline$>6$ mos old & 2 \\
\hline \multicolumn{2}{|c|}{$\begin{array}{l}\text { Surgical option preferred by surgeon when } \\
\text { child aged }\end{array}$} \\
\hline \multicolumn{2}{|l|}{$<3$ mos old } \\
\hline SCS & 10 \\
\hline \multicolumn{2}{|l|}{ Vault } \\
\hline Either & 2 \\
\hline \multicolumn{2}{|l|}{$3-6$ mos old } \\
\hline SCS & 7 \\
\hline \multicolumn{2}{|l|}{ Vault } \\
\hline Either & 5 \\
\hline \multicolumn{2}{|l|}{$>6$ mos old } \\
\hline \multicolumn{2}{|l|}{ SCS } \\
\hline Vault & 12 \\
\hline Either & \\
\hline
\end{tabular}

only 2/12 surgeons include SCS in the discussion. The surgeons' procedure of choice is usually SCS (10/12) for children presenting at an age $<3$ months; the other 2 surgeons expressed no preference and give the family the option of SCS or open surgery. For patients between 3 and 6 months of age, 5 surgeons give the family both options and let them choose, whereas 7 surgeons still prefer SCS ( 1 of whom prefers springs). For patients over 6 months of age, all surgeons prefer open vault surgery.

\section{Patient Preparation}

All surgeons do a skin preparation that includes povidone-iodine. Six use it alone, 5 use a chlorhexidine scrub before using povidone-iodine, and 1 uses a povidone-iodine scrub followed by alcohol and then a povidone-iodine preparation. The mode of skin preparation is the same for SCS and open vault surgery. All surgeons use prophylactic cefazolin (for both types of procedures; Table 4); 9/12 use a dose of $25-30 \mathrm{mg} / \mathrm{kg}$ and 3 surgeons (1 site) use 50 $\mathrm{mg} / \mathrm{kg}$. Arterial lines are universally used in open vault surgery (12/12) and are also commonly used for strip surgery (9/11 surgeons who have performed SCS recently). A Foley catheter is also universally used in open vault cases (12/12), but only 4/11 surgeons place one for SCS cases. Precordial Doppler is used by half of the surgeons for both types of surgery. Tranexamic acid, including a loading dose and continuous dosing to the end of the case, is routinely used by all surgeons at 3 sites ( 7 surgeons) for both types of surgery; the other 2 sites (5 surgeons) do not use tranexamic acid for any cases. Preoperative blood work for cranial vault surgery includes complete blood count and cross-match for 9 surgeons, 1 surgeon does a cross-match in the operating room, and 2 surgeons do no preoperative
TABLE 4. Perioperative adjuncts

\begin{tabular}{lcc}
\hline \multirow{2}{*}{ Step } & \multicolumn{2}{c}{ No. of Surgeons } \\
\cline { 2 - 3 } & SCS $\left(\mathrm{n}=11^{*}\right)$ & Cranial Vault $(\mathrm{n}=12)$ \\
\hline Cefazolin prior to incision & 11 & 12 \\
\hline Arterial line & 9 & 12 \\
\hline Foley catheter & 4 & 12 \\
\hline Precordial Doppler & 6 & 6 \\
\hline Tranexamic acid & 7 & 7 \\
\hline
\end{tabular}

$n=$ total number of surgeons.

* One surgeon had not performed SCS in the preceding year and therefore did not answer questions related to the procedure.

blood work. For SCS, 8/11 surgeons get a preoperative complete blood count and 6/11 cross-match blood.

\section{Strip Craniectomy Technique}

Eleven surgeons described their technique for performing SCS: one had not done such a procedure in the preceding year and therefore did not answer these questions. Two surgeons (2/11, one site) perform an open strip craniectomy, $2 \mathrm{~cm}$ wide, without endoscopic assistance via a lazy-S incision. They place distracting springs and do not use a postoperative drain or helmet but do use a wound dressing. Their patients do not go to the intensive care unit, and they expect patients to go home on postoperative day 1.

The other 9 surgeons prefer endoscope-assisted craniectomy followed by helmet therapy (Table 5). All 9 perform this procedure via 2 transverse incisions, each measuring 2-4 cm (mean $3.1 \mathrm{~cm}$ ) in length. The craniectomy measures $2-5 \mathrm{~cm}$ in width (mean $4.3 \mathrm{~cm}, 6 / 9$ surgeons do

TABLE 5. Characteristics of endoscope-assisted SCS procedures

\begin{tabular}{lc}
\hline \multicolumn{1}{c}{ Step } & No. of Surgeons $(\mathrm{n}=9)$ \\
\hline Patient position & 5 \\
\hline Sphinx & 3 \\
\hline Prone & 1 \\
\hline Supine & 2 \\
\hline Craniectomy width & 1 \\
\hline $2 \mathrm{~cm}$ & 6 \\
\hline $2-5 \mathrm{~cm}$ & 7 \\
\hline $5 \mathrm{~cm}$ & \\
\hline Parietal wedge osteotomy & 9 \\
\hline Endoscope use & 5 \\
\hline Epidural dissection & 0 \\
\hline Bone cautery & 4 \\
\hline Postop care & \\
\hline Subgaleal drain & $3(1$ site $)$ \\
\hline Intensive care unit stay & $4(2$ sites $)$ \\
\hline CT
\end{tabular}

$F U=$ follow-up. 
TABLE 6. Characteristics of cranial vault surgery

\begin{tabular}{lc}
\hline \multicolumn{1}{c}{ Step } & No. of Surgeons $(n=12)$ \\
\hline Patient position & \\
\hline Sphinx & 4 \\
\hline Prone & 5 \\
\hline Either & 3 \\
\hline Forehead remodeled & 9 \\
\hline Preferred procedure & 3 \\
\hline$\quad$ Modified Pi w/o forehead remodeling & 3 \\
\hline Total vault w/ forehead remodeling & 2 \\
\hline Ant 2/3 vault w/ forehead remodeling & 4 \\
\hline No strong preference & \\
\hline CT & $5(2$ sites $)$ \\
\hline Prior to discharge & $4(2$ sites $)$ \\
\hline In 1st-yr FU
\end{tabular}

Ant $=$ anterior.

a craniectomy that is $5 \mathrm{~cm}$ wide). In addition, wedge osteotomies are created in the parietal bones by $7 / 9$ surgeons (6 surgeons perform 2 wedges in each parietal bone, and 1 surgeon makes a single wedge osteotomy). One surgeon adds a wedge osteotomy in the occipital bone. All 9 surgeons use the endoscope for the epidural dissection (8 use the $30^{\circ}$ scope and 1 uses the $0^{\circ}$ scope), and 5 surgeons use a scope to do cautery on the bone edge. None use a postoperative subgaleal drain, 1 uses a dressing, and 7/9 apply antibiotic ointment to the wounds. Four surgeons prefer to have their patients in the intensive care unit after surgery. All surgeons expect their SCS patients to go home on postoperative day 1 or 2 . At 1 site (3 surgeons), patients routinely undergo CT prior to discharge. All 9 surgeons have their patients use helmets after endoscope-assisted craniectomy; they instruct patients to wear the helmet for 22-23.5 hours/day and expect helmet therapy to continue for 6-12 months (mean 7.3 months).

Follow-up evaluations in the clinic after SCS are done by plastic surgery alone at 1 site; at all other sites, the patients see both plastic surgery and neurosurgery in the follow-up. At the follow-up evaluations, 8 surgeons measure occipital frontal circumference, 6 measure cephalic index, and 5 obtain a laser scan. At 2 sites (4 surgeons), routine follow-up includes a CT scan within the 1st year.

\section{Cranial Vault Surgery}

For cranial vault surgery for isolated sagittal synostosis (Table 6), all surgeons use a bicoronal incision. The preferred patient position is prone for 5 surgeons, sphinx for 4 surgeons, and either supine or prone for 3 surgeons. The surgical technique varied according to the deformity, but $3 / 12$ surgeons usually prefer a modified Pi procedure without forehead remodeling, 3 others prefer a total cranial vault procedure with forehead remodeling, 2 prefer an anterior $2 / 3$ cranial vault procedure with forehead remodeling, and 4 surgeons did not express a preference. Nine surgeons include the forehead in the repair. Defects are managed with particulate bone graft (7/12), split thickness bone graft (2/12), or a combination of the two (3/12). Bone fixation is done with absorbable plates (6 surgeons) or a combination of suture and absorbable plates (6 surgeons). Eleven surgeons use a subgaleal drain and 9/12 use a head dressing. All apply antibiotic ointment to the wound, and all send their patients to the intensive care unit after surgery. Two sites (5 surgeons) routinely do a CT scan prior to discharge. Patients are expected to go home on postoperative days 3-5 (mean 3.7 days). Follow-up evaluations in the clinic after cranial vault surgery are done by plastic surgery alone at 1 site; at all other sites, the patients see both plastic surgery and neurosurgery in the follow-up. At follow-up evaluations, 7 surgeons measure occipital frontal circumference, 4 measure cephalic index, and $1 \mathrm{ob}-$ tains a laser scan. At 2 sites (4 surgeons), routine follow-up includes a CT scan within the 1st year.

\section{Discussion}

Multicenter groups have the potential to generate large patient numbers, collect accurate data prospectively, and answer important questions in clinical medicine. We created the SynRG in 2016 to contribute multicenter data to the care of patients with craniosynostosis. As part of the network launch, we surveyed SynRG surgeons about sagittal synostosis to identify areas of practice that we have in common and areas in which we differ. From these results, we are able to discern topics for further study and assess the feasibility of developing common protocols.

We found significant variation in a number of areas including methods of patient assessment, imaging, and surgical approach. Variation in the management of sagittal synostosis has been identified by others. ${ }^{3}$ In contrast to our survey results, Doumit et al. found that only $35 \%$ of surgeons preferred an endoscopic approach in children younger than 4 months of age and that $71 \%$ of surgeons preferred to have CT done regardless of the mode of presentation. ${ }^{3}$ Some of the differences between their results and ours may be attributable to their inclusion of surgeons from multiple countries.

The SynRG surgeons varied in their choice of surgical approach. Endoscopic craniectomy followed by helmet therapy was preferred over cranial vault surgery in as few as $20 \%$ to as many as $83 \%$ of patients. Both of these techniques are in common clinical use for children who present in the first few months of life. The choice between these two procedures before the patient reaches 6 months of age is likely the biggest question that is currently unanswered in this condition. A study randomizing patients between these procedures may be challenging but should be considered. Other study designs with accurate prospective data collection and an objective outcome measure could also provide valuable information and may be more feasible. ${ }^{8,10}$

The use of CT also appears to be quite variable among members of the SynRG. Three sites include CT in their preoperative evaluation. Data on radiation exposure in infants suggest that the risk may not be zero. ${ }^{1}$ In addition, if sedation is required, there may be additional risk. ${ }^{4,6} \mathrm{On}$ the other hand, confirming the diagnosis via CT in questionable cases allows for a more accurate discussion with families. Investigation of the use of preoperative imaging 
and perhaps the development of guidelines for the selective use of scanning seems appropriate. Ultrasound has been reported as a useful method of assessing suture patency ${ }^{9}$ and perhaps deserves assessment in a larger number of children.

Among the 9 surgeons who regularly perform endoscopic SCS, there was substantial variation in the amount of bone removal. This issue was evaluated in an agematched study by Dlouhy et al. in St Louis. ${ }^{2}$ They found no apparent difference in the cephalic index at 1 year between a 2-cm craniectomy without parietal osteotomies and a $5-\mathrm{cm}$ craniectomy with parietal osteotomies. Their study suggests that the smaller bone removal may be adequate. This important question should be assessed in a larger randomized trial including multiple sites and surgeons using the outcomes of cranial shape, presence of cranial defect requiring repair, and intraoperative blood loss.

Four of our surgeons prefer to have their SCS patients in the intensive care unit after surgery. Detailed prospective data collection may allow us to determine whether this practice prevents postoperative complications and justifies the added cost. Similarly, two of the SynRG sites perform CT scanning prior to discharge and/or at 1 year postoperatively. Again, considering the radiation exposure and additional costs, we need to assess the risk/benefit ratio of this practice.

There is now a substantial body of quality improvement literature in which standardized protocols are used in surgery to reduce practice variation and improve outcomes. ${ }^{5,7}$ In light of the practice variation observed within the SynRG, there appears to be a large opportunity to implement quality improvement methods in the care of children with craniosynostosis. Our survey did not look at patient outcomes. Certainly, the variation we observed should be compared with outcomes, and this will be a focus of future SynRG work using our multicenter database.

This survey may not represent practice patterns across the specialty since it only included 5 sites. With a broader survey, we might observe even more variation, but we cannot conclude that from our relatively small sample. Our intent, though, was not to assess the national practice pattern. Our intent was to assess the practice within our network to identify opportunities for research and improvement. We believe this should begin with a small group of interested sites, with the potential to grow in the future. Although limited in scope to our small group, the results highlight the degree of baseline variability in patient management and call attention to the opportunities that exist if the group can transcend this variability to address research questions in the craniosynostosis population.

\section{Conclusions}

In summary, it is our belief that there are many important unanswered questions in the management of craniosynostosis. On the basis of the experience of other multicenter collaborative groups, we believe that such a process will give us the best chance of contributing important knowledge to the field. We, therefore, think it is worthwhile continuing to build our network and multicenter database to answer these important questions.

\section{Acknowledgments} paper.

We thank Kristin Kraus, MSc, for editorial assistance with this

\section{References}

1. Berrington de Gonzalez A, Salotti JA, McHugh K, Little MP, Harbron RW, Lee C, et al: Relationship between paediatric CT scans and subsequent risk of leukaemia and brain tumours: assessment of the impact of underlying conditions. $\mathbf{B r}$ J Cancer 114:388-394, 2016

2. Dlouhy BJ, Nguyen DC, Patel KB, Hoben GM, Skolnick GB, Naidoo SD, et al: Endoscope-assisted management of sagittal synostosis: wide vertex suturectomy and barrel stave osteotomies versus narrow vertex suturectomy. J Neurosurg Pediatr 25:674-678, 2016

3. Doumit GD, Papay FA, Moores N, Zins JE: Management of sagittal synostosis: a solution to equipoise. J Craniofac Surg 25:1260-1265, 2014

4. Glatz P, Sandin RH, Pedersen NL, Bonamy AK, Eriksson LI, Granath F: Association of anesthesia and surgery during childhood with long-term academic performance. JAMA Pediatr 171:e163470, 2017

5. Haynes AB, Weiser TG, Berry WR, Lipsitz SR, Breizat AH, Dellinger EP, et al: A surgical safety checklist to reduce morbidity and mortality in a global population. N Engl J Med 360:491-499, 2009

6. Ing C, DiMaggio C, Whitehouse A, Hegarty MK, Brady J, von Ungern-Sternberg BS, et al: Long-term differences in language and cognitive function after childhood exposure to anesthesia. Pediatrics 130:e476-e485, 2012

7. Kestle JR, Holubkov R, Douglas Cochrane D, Kulkarni AV, Limbrick DD Jr, Luerssen TG, et al: A new Hydrocephalus Clinical Research Network protocol to reduce cerebrospinal fluid shunt infection. J Neurosurg Pediatr 17:391-396, 2016

8. Kulkarni AV, Drake JM, Kestle JR, Mallucci CL, Sgouros S, Constantini S: Endoscopic third ventriculostomy vs cerebrospinal fluid shunt in the treatment of hydrocephalus in children: a propensity score-adjusted analysis. Neurosurgery 67:588-593, 2010

9. Proisy M, Riffaud L, Chouklati K, Tréguier C, Bruneau B: Ultrasonography for the diagnosis of craniosynostosis. Eur J Radiol 90:250-255, 2017

10. Walicke P, Abosch A, Asher A, Barker FG II, Ghogawala Z, Harbaugh R, et al: Launching effectiveness research to guide practice in neurosurgery: a National Institute Neurological Disorders and Stroke Workshop report. Neurosurgery 80:505-514, 2017

\section{Disclosures}

Dr. Patel has been a consultant for Stryker CMF.

\section{Author Contributions}

Conception and design: all authors. Acquisition of data: all authors. Analysis and interpretation of data: all authors. Drafting the article: Kestle. Critically revising the article: all authors. Reviewed submitted version of manuscript: all authors. Approved the final version of the manuscript on behalf of all authors: Kestle.

\section{Correspondence}

John R. W. Kestle: Primary Children's Hospital, University of Utah, Salt Lake City, UT. neuropub@hsc.utah.edu. 